\title{
EQUAÇÕES DIFERENCIAIS PARCIAIS: ESTUDO DE CASO
}

\author{
Bruno Claudino dos Santos, Viviane Colucci, Vitória Maria Almeida Teodoro de Oliveira, Felipe \\ Borino Giroldo, Leticia Darlla Cordeiro. \\ Universidade Tecnológica Federal do Paraná - UTFPR, Engenharia Eletrônica, Campo Mourão, Paraná. Email: \\ bsantos@alunos.utfpr.edu.br. \\ Agência de fomento: Universidade Tecnológica Federal do Paraná
}

\section{RESUMO}

Existem muitas ferramentas matemáticas de grande importância e utilidade relacionadas a modelagem matemática, que podem ser utilizadas em muitos tipos de situações quando se trata de solucionar diferentes problemas e situações nas mais diversas áreas conhecidas pelo homem. 0 objetivo desse trabalho é apresentar a solução de um problema de valor de contorno, contendo um caso particular de uma equação diferencial parcial do calor, utilizando para isto, algumas ferramentas matemáticas conhecidas, dentre elas o método da separação de variáveis, a transformada de Laplace e a série de Fourier. Essas ferramentas serão aplicadas no estudo de caso citado anteriormente e seu resultado analisado para garantir uma solução satisfatória.

Palavras-chave: Série de Fourier, equações diferenciais parciais, fenômenos físicos, modelagem matemática, equação do calor.

\section{PARTIAL DIFFERENTIAL EQUATIONS: CASE STUDY}

\begin{abstract}
There are many mathematical tools of great importance and usefulness related to mathematical modeling, which can be used in many types of situations when it comes to solving different problems and situations in the most diverse areas known to man. The objective of this work is to present the solution of a contour value problem, containing a particular case of a partial differential heat equation, using for this purpose some known mathematical tools, among them the method of separation of variables, the Laplace transform And the Fourier series. These tools will be applied in the case study cited above and its result analyzed to ensure a satisfactory solution.

Keywords: Fourier series, partial differential equations, physical phenomena, mathematical modeling, heat equation.
\end{abstract}




\section{INTRODUÇÃO}

Existem muitas técnicas tradicionais para solucionar um determinado problema matemático, sendo que muitas vezes representar um fenômeno físico por meio de técnicas analíticas não é tão simples.

O uso de leis da física possibilita obter equações diferenciais que representam os fenômenos em estudo. $O$ sucesso em tratar esses problemas utilizando o Cálculo Diferencial foi um enorme estímulo aos físicos e matemáticos do século XVIII, incentivando a busca de modelos para diversos problemas da Mecânica do Contínuo e de outros ramos da Física que expressassem os fenômenos em termos de Equações Diferenciais (FIGUEIREDO, 2012).

Utiliza-se a modelagem matemática para representar esses fenômenos geralmente por meio de equações, em destaque as equações diferenciais parciais. Segundo Bassanezi (1994, p. 31) essa modelagem se reduz quase sempre a um sistema de equações ou inequações algébricas, diferenciais, integrais, etc., obtido por meio de relações estabelecidas entre as variáveis consideradas essenciais ao fenômeno sobre análise.

Ao longo de muitos anos diversos casos particulares de equações diferenciais parciais foram solucionados por meio de métodos analíticos e numéricos sujeitos a diferentes condições. Para auxiliar na tentativa de solução dos mais variados problemas foram desenvolvidos diferentes métodos matemáticos, entre eles, a série de Fourier, que será o foco deste trabalho.

\section{METODOLOGIA}

Por meio da série de Fourier é possível solucionar diversos tipos de problemas, entre eles, equações diferenciais parciais, mas para isso é necessário expressar uma função dada como uma série de senos e/ou cossenos. Seguindo esse raciocínio, a série de Fourier pode ser definida da seguinte forma.

Seja $f: R \rightarrow R$ uma função periódica de período $2 L$, integrável e absolutamente integrável em cada intervalo limitado. Em particular, o resultado de $\int_{-L}^{L}|f(x)| d x$ é um número finito.

A série de Fourier é dada pela equação (1) e as equações (2), (3) e (4) são respectivamente seus coeficientes $a_{0}, a_{n}$ e $b_{n}$. Os números $a_{n}$ para $n \geq 0$ e $b_{n}$ para $n \geq 1$ são definidos como os coeficientes da série de Fourier da função $f$.

$$
\begin{gathered}
f(x) \approx \frac{a_{0}}{2}+\sum_{n=1}^{\infty}\left(a_{n} \cos \frac{n \pi x}{L}+b_{n} \sin \frac{n \pi x}{L}\right) \\
a_{0}=\frac{1}{L} \int_{-L}^{L} f(x) d x \\
a_{n}=\frac{1}{L} \int_{-L}^{L} f(x) \cos \frac{n \pi x}{L} d x \\
b_{n}=\frac{1}{L} \int_{-L}^{L} f(x) \operatorname{sen} \frac{n \pi x}{L} d x .
\end{gathered}
$$

Essas definições possibilitam a aplicação e resolução de diversas equações diferenciais parciais, como exemplos, a equação de condução de calor em uma barra, a equação de Laplace, a equação da onda e etc.

Esse método será utilizado para solucionar o problema de valor de contorno

$$
\left\{\begin{array}{cc}
\frac{\partial u}{\partial t}=3 \frac{\partial^{2} u}{\partial x^{2}}, & t>0,0<x<2 \\
u(0, t)=2(u, t)=0, & t>0 \\
u(x, 0)=x, & 0<x<2 .
\end{array}\right.
$$




\section{RESULTADOS}

Para encontrar uma solução para (5) é necessário primeiramente aplicar o método da separação de variáveis. Seja

$$
u(x, t)=X(x) T(t)
$$

Ao substituir (6) em (5)

$$
\begin{aligned}
& X \frac{\partial T}{\partial t}=3 T \frac{\partial^{2} X}{\partial x^{2}} \\
& \frac{1}{3 T} \frac{\partial T}{\partial t}=\frac{1}{X} \frac{\partial^{2} X}{\partial x^{2}}=-\lambda^{2} .
\end{aligned}
$$

Da equação (7) obtém-se as equações diferenciais ordinárias

$$
\begin{gathered}
\frac{d T}{d t}+3 \lambda^{2} T=0 \\
\frac{d^{2} X}{d x^{2}}+\lambda^{2} X=0 .
\end{gathered}
$$

Solucionando a equação (8) por métodos tradicionais obtém-se

$$
T=c e^{-3 \lambda^{2} t}
$$

Solucionando (9) por meio da Transformada de Laplace,

$$
\begin{aligned}
& \mathcal{L}\left[\frac{d T}{d t}+\lambda^{2} X\right]=\mathcal{L}[0] \\
& \mathcal{L}[X]=\frac{S X(0)}{S^{2}+\lambda^{2}}+\frac{X^{\prime}(0)}{S^{2}+\lambda} .
\end{aligned}
$$

Aplicando a Transformada de Laplace inversa em (11) tem-se

$$
\mathcal{L}^{-1}\left[\frac{X^{\prime}(0)}{s^{2}+\lambda^{2}}\right]=\frac{X^{\prime}(0)}{\lambda} \sin \lambda x .
$$

Sendo as constantes

$$
\begin{aligned}
& X(0)=C_{1} \\
& \frac{X^{\prime}(0)}{\lambda}=C_{2} .
\end{aligned}
$$

Ao substituir (12), (13) e (14) em (11),

$$
X=C_{1} \cos \lambda x+C_{2} \sin \lambda x \text {. }
$$

Se substituir as soluções encontradas (10) e (15) em (6), obtém-se

$$
\begin{aligned}
& u(x, t)=\left[C_{1} \cos \lambda x+C_{2} \sin \lambda x\right] C e^{-3 \lambda^{2} t} \\
& u(x, t)=[A \cos \lambda x+B \sin \lambda x] e^{-3 \lambda^{2} t}
\end{aligned}
$$

Aplicando a condição de contorno $u(0, t)=u(2, t)=0, t>0$ em (16) encontra-se as constantes $A$ e $B$, descritas respectivamente nas equações (17) e (18).

$$
\begin{gathered}
u(0, t)=[A \cos \lambda 0+B \sin \lambda 0] e^{-3 \lambda^{2} t}=0 \\
u(0, t)=A e^{-3 \lambda^{2} t}=0 \\
A=0 \\
u(2, t)=[A \cos \lambda 2+B \sin \lambda 2] e^{-3 \lambda^{2} t}=0 \\
u(2, t)=[0 \cos \lambda 2+B \sin \lambda 2] e^{-3 \lambda^{2} t}=0 \\
B=0 .
\end{gathered}
$$

Nota-se que $B=0$ satisfaz a condição de contorno, então torna-se trivial encontrar o valor de $\lambda$. 


$$
\begin{gathered}
u(2, t)=B \sin (2 \lambda) e^{-3 \lambda^{2} t} \\
\sin 2 \lambda=0 \\
2 \lambda=n \pi \\
\lambda=\frac{n \pi}{2}, n \in \mathbb{Z} .
\end{gathered}
$$

Quanto ao valor encontrado $A=0$, ao substitui-lo em (16) terá

Aplicando (19) em (20)

$$
u(x, t)=B \sin (\lambda x) e^{-3 \lambda^{2} t} .
$$

$$
u(x, t)=B e^{-\frac{3 n^{2} \pi^{2} t}{4}} \sin \frac{n \pi x}{2}
$$

Para indicar que diferentes constantes podem ser utilizadas para diferentes valores de $n$ e seguindo o princípio da superposição no qual as somas das soluções de (21) também são soluções, então é possível reescrever

$$
u(x, t)=\sum_{n=1}^{\infty} B_{n} e^{-\frac{3 n^{2} \pi^{2} t}{4}} \sin \frac{n \pi x}{2} .
$$

Para finalizar, (22) precisa satisfazer a outra condição inicial $u(x, 0)=x, 0<x<2$, sendo assim, substituindo $t=0$,

$$
\begin{aligned}
u(x, 0) & =\sum_{n=1}^{\infty} B_{n} e^{-\frac{3 n^{2} \pi^{2} 0}{4}} \sin \frac{n \pi x}{2}=x \\
x & =\sum_{n=1}^{\infty} B_{n} \sin \frac{n \pi x}{2}, \quad 0<x<2 .
\end{aligned}
$$

Para expandir $f(x)=x,-2<x<2$, em uma série de Fourier é necessário encontrar primeiramente seus coeficientes,

$$
\begin{gathered}
a_{0}=\frac{1}{2} \int_{-2}^{2} x d x=0 \\
a_{n}=\frac{1}{2} \int_{-2}^{2}\left[x \cos \left(\frac{n \pi x}{2}\right)\right] d x=0 \\
b_{n}=\frac{1}{2} \int_{-2}^{2}\left[x \sin \left(\frac{n \pi x}{2}\right)\right] d x=-\frac{4}{n \pi} \cos n \pi .
\end{gathered}
$$

Aplicando os coeficientes encontrados

$$
x=\sum_{n=1}^{\infty} b_{n} \sin \frac{n \pi x}{2} .
$$

Percebe-se que a série de Fourier encontrada é uma série de senos equivalente a série encontrada em (23) e como $b_{n}$ é diferente de zero é possível reescrever da seguinte forma

$$
\begin{gathered}
b_{n}=-\frac{4}{n \pi}(-1)^{n} \\
b_{n}=\frac{4}{n \pi}(-1)^{n+1} .
\end{gathered}
$$

Substituindo (28) em (22) pela equivalência citada anteriormente, tem-se

$$
u(x, t)=\sum_{n=1}^{\infty} \frac{4}{n \pi}(-1)^{n+1} e^{-\frac{3 n^{2} \pi^{2} t}{4}} \sin \frac{n \pi x}{2}
$$




$$
u(x, t)=\frac{4}{\pi} \sum_{n=1}^{\infty} \frac{(-1)^{n+1}}{n} e^{-\frac{3 n^{2} \pi^{2} t}{4}} \sin \frac{n \pi x}{2}
$$

Assim, a equação (29) é a solução do problema de valor de contorno proposto em (5).

As figuras (1) e (2) demonstram as soluções, respectivamente, através da série de Fourier e para fins de comparações, pelo Método das Diferenças Finitas, obtidas aplicando os resultados através de um algoritmo desenvolvido no software Matlab ${ }^{\circledR}$.

Figura 1. Solução da EDP pelo método da série de Fourier.

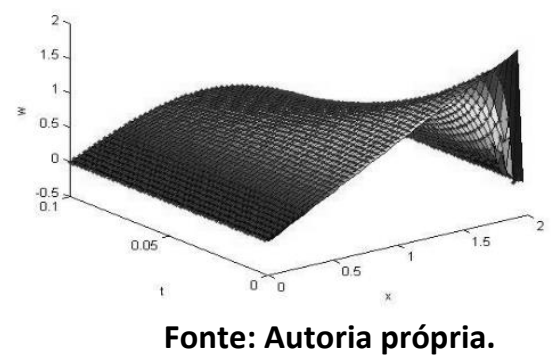

Figura 2. Solução da EDP pelo Método das Diferenças Finitas.

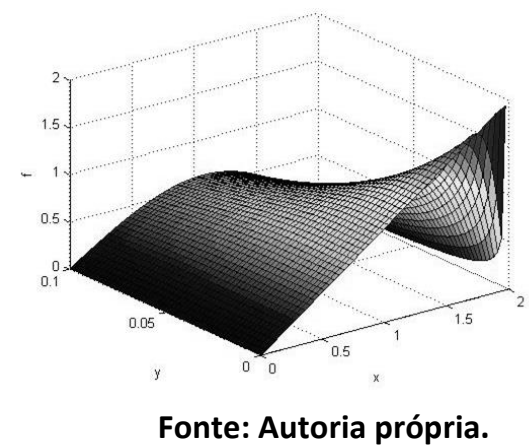

\section{DISCUSSÃO}

Percebe-se que a série de Fourier proporcionou uma solução ao problema, demonstrando assim, que este método tem grande utilidade, devido ao fato de poder ser aplicado nas mais diversas áreas e situações e támbém por suas características, podendo a série ser descrita na forma trigonométrica, harmônica e complexa. Algumas vezes a solução encontrada não é a forma esperada pois seu resultado não é tão trivial. Embora seja necessário um certo nível de abstração a solução é interessante pois em muitos casos não é possível resolver uma equação diferencial parcial por métodos analíticos.

Para um melhor entendimento, o resultado aproximado pode ser obtido aplicando o resultado encontrado em algoritmos e softwares computacionais.

\section{CONCLUSÃO}

A solução do problema de valor de contorno utilizando a série de Fourier conforme apresentada neste trabalho foi comparada com uma solução encontrada do mesmo problema através do Método das diferenças Finitas para garantir que a solução é satisfatória, conforme demonstrado anteriormente nas figuras (1) e (2). Com isto, pode-se observar a proximidade entre elas e assim, validar o resultado discutido neste trabalho.

Este estudo de caso foi desenvolvido em um projeto de extensão na Universidade Tecnológica Federal do Paraná, campus Campo Mourão. 


\section{REFERÊNCIAS}

BASSANEZI, R. Modelagem Matemática. Dynamis, Blumenau, v. 1, n. 7, abr./jun. 1994. p. 55-83.

FIGUEIREDO, D.G. Análise de Fourier e Equações Diferenciais Parciais. Rio de Janeiro: IMPA, 2007.

IÓRIO, Valéria de Magalhães. EDP um curso de graduação. 3. ed. Rio de Janeiro, RJ: IMPA, 2012. p. 275. 